report demonstrated that the use of insecticide to impregnate bed nets and screening for parasites in blood reduced the malaria risk in children on iron supplementation. ${ }^{17}$ Moreover, recent data demonstrate that iron deficiency negatively impacts on immunological responses to diphtheria vaccine leaving children insufficiently protected against such infections ( $N$ Stoffel, Zurich, oral presentation, Bioiron Meeting 2019). Thus, this study by Armitage ${ }^{15}$ and co-workers is an important step forward to gain more insights into the relative contribution of different regulatory mechanisms on circulating biomarker concentrations such as hepcidin and how this impacts on predicting therapeutic efficacy and the risk:benefit ratio of iron supplementation in a primary care setting.

Future studies will have to clarify the optimal timing and dose of iron supplementation to children, whether or not a continuous administration via dietary iron fortification or a once daily or once every other day application is preferable. ${ }^{18}$ It will also be necessary to identify those children who might be at risk of unwanted effects of iron supplementation mainly arising from an increased morbidity and mortality from infections. Finally, we await further information on the impact of iron supplementation on growth and mental development, functionality of the immune system, efficacy of preventive measures such as vaccination, and the consequences of iron-mediated alterations of the intestinal microbiota on children's health.

\section{References}

1. Muckenthaler MU, Rivella S, Hentze MW, Galy B. A Red Carpet for Iron Metabolism. Cell. 2017;168(3):344-361.

2. Camaschella C. Iron deficiency. Blood. 2019;133(1):30-39.

3. Weiss G, Carver PL. Role of divalent metals in infectious disease susceptibility and outcome. Clin Microbiol Infect. 2018;24(1):16-23.
4. Armitage AE, Drakesmith H. Genetics. The battle for iron. Science. 2014;346(6215):1299-1300.

5. Soares MP, Weiss G. The Iron age of host-microbe interactions. EMBO Rep. 2015;16(11):1482-1500.

6. Gwamaka M, Kurtis JD, Sorensen BE, et al. Iron deficiency protects against severe Plasmodium falciparum malaria and death in young children. Clin Infect Dis. 2012;54(8):1137-1144

7. Girelli D, Nemeth E, Swinkels DW. Hepcidin in the diagnosis of iron disorders. Blood. 2016;127(23):2809-2813.

8. Theurl I, Aigner E, Theurl M, et al. Regulation of iron homeostasis in anemia of chronic disease and iron deficiency anemia: diagnostic and therapeutic implications. Blood. 2009;113(21):5277-5286.

9. Paganini D, Uyoga MA, Zimmermann MB. Iron Fortification of Foods for Infants and Children in Low-Income Countries: Effects on the Gut Microbiome, Gut Inflammation, and Diarrhea. Nutrients. 2016;8(8).

10. Prentice AM, Doherty CP, Abrams SA, et al. Hepcidin is the major predictor of erythrocyte iron incorporation in anemic African children. Blood. 2012;119(8):1922-1928.

11. Weiss G, Ganz T, Goodnough LT. Anemia of inflammation. Blood. 2019;133(1):40-50.

12. Theurl I, Schroll A, Nairz M, et al. Pathways for the regulation of hepcidin expression in anemia of chronic disease and iron deficiency anemia in vivo. Haematologica. 2011;96(12):1761-1769.

13. Stoffel NU, Lazrak M, Bellitir S, et al. The opposing effects of acute inflammation and iron deficiency anemia on serum hepcidin and iron absorption in young women. Haematologica. 2019 Jan 10. [Epub ahead of print]

14. Reinisch W, Staun M, Tandon RK, et al. A randomized, open-label, non-inferiority study of intravenous iron isomaltoside 1,000 (Monofer) compared with oral iron for treatment of anemia in IBD (PROCEED). Am J Gastroenterol. 2013;108(12):1877-1888.

15. Armitage AE, Agbla SC, Betts M, et al. Rapid growth is a dominant predictor of hepcidin suppression and declining ferritin in Gambian infants. Haematologica. 2019;104(8):1542-1553.

16. Andrews NC. Genes determining blood cell traits. Nat Genet. 2009;41(11):1161-1162.

17. Aimone AM, Brown P, Owusu-Agyei S, Zlotkin SH, Cole DC. Impact of iron fortification on the geospatial patterns of malaria and nonmalaria infection risk among young children: a secondary spatial analysis of clinical trial data from Ghana. BMJ Open. 2017;7(5):e013192.

18. Stoffel NU, Cercamondi CI, Brittenham G, et al. Iron absorption from oral iron supplements given on consecutive versus alternate days and as single morning doses versus twice-daily split dosing in iron-depleted women: two open-label, randomised controlled trials. Lancet Haematol. 2017;4(11):e524-e533.

\title{
The wolf of hypomethylating agent failure: what comes next?
}

\author{
Anne Sophie Kubasch ${ }^{1,2,3}$ and Uwe Platzbecker ${ }^{1,2,3}$ \\ ${ }^{1}$ Medical Clinic and Policlinic 1, Hematology and Cellular Therapy, Leipzig University Hospital, Germany; ${ }^{2}$ German MDS Study \\ Group (G-MDS) and ${ }^{3}$ European Myelodysplastic Syndromes Cooperative Group (EMSCO group, www.emsco.eu) \\ E-mail: UWE PLATZBECKER - uwe.platzbecker@medizin.uni-leipzig.de
}

doi:10.3324/haematol.2019.222794

M yelodysplastic syndromes (MDS) and acute myeloid leukemia (AML) are clonal hematopoietic stem/progenitor cell (HSPC) disorders mainly affecting the elderly population. ${ }^{1}$ Hypomethylating agents (HMA) like azacitidine and decitabine have become the standard of care in elderly patients with highrisk (HR) MDS or AML unfit for intensive treatment approaches. Until today, responses to HMA have occured in less than $50 \%$ of patients and are not durable, with only a few patients achieving long-lasting remissions. ${ }^{2,3}$ Prognostic clinical markers, such as presence of peripheral blasts, high transfusion burden, and poor performance status, have been identified as indicators of a worse outcome of HMA-based therapy., ${ }^{1,4}$ Moreover, responses to HMA are especially short-lived in patients with adverse risk cytogenetic abnormalities compared to those with normal karyotype. ${ }^{1}$

Craddock et al. evaluated the impact of mutational profile on clinical response to azacitidine by analyzing 250 patients with newly diagnosed, relapsed, or refractory AML or HR-MDS. Lower complete response (CR) rates occurred in patients with an IDH2 and STAG2 mutation, higher CR rates in patients with NPM1 mutation. Mutations in CDKN2A, IDH1, TP53, NPM1, and FLT3ITD were associated with a worse overall survival (OS) in univariate analysis, while multivariate analysis showed a decrease in OS in patients with CDKN2A, IDH1, or TP53 mutations. Moreover, ASXL1 and ETV6 were associated with short response duration after azacitidine treatment. ${ }^{5}$

Despite all efforts to try to select patients based on 
their cytogenetic and molecular characteristics, failing HMA therapy is still associated with a dismal prognosis reflected by a median survival of six months. ${ }^{6}$ Until now, almost nothing has been known about the mechanisms underlying HMA-resistance. Thus, as frequently as possible, patients experiencing HMA failure should be evaluated for clinical trial options, given the current absence of any available standard treatment in that setting. In clinically fit patients with HR-MDS or secondary AML (sAML) and normal karyotype, intensive chemotherapy with a subsequent allogeneic stem cell transplantation may also be considered.

In this issue of the Journal, Sébert et al. report results of a phase II study of the Groupe Francophone des Myélodysplasies (GFM) investigating the novel HMA guadecitabine (SGI-110) as a salvage treatment in HRMDS and low blast count AML ( $<30 \%$ bone marrow blasts) patients after azacitidine failure. Guadecitabine is a dinucleotide of decitabine and deoxyguanosine with similar potency but longer half life due to resistance to cytidine deaminase degradation. This results in an extended exposure of blasts to its active metabolite decitabine. The study included fifty-six patients (median age 75 years) who failed or relapsed after at least six previous azacitidine cycles. Patients' characteristics indicated a study population with advanced disease, including $87.5 \%$ of patients carrying high-risk somatic mutations such as ASXL1 (25\%), RUNX1 (21\%), TP53 (20\%) and U2AF1 (20\%). Patients achieving hematologic response after 3,6 or 9 cycles of guadecitabine $\left(60 \mathrm{mg} / \mathrm{m}^{2} /\right.$ day subcutaneously days $1-5$ of 28 -day treatment cycles) were considered to be responders and were allowed to continue treatment until loss of response. Sébert et al. identified eight $(14.3 \%)$ responding patients, including two CR, one partial response (PR), three hematologic improvements
(HI), and two marrow CR (mCR). Median response duration was 11.5 months and median OS 7.1 months; responders had a prolonged median OS of 17.9 months.?

Therefore, even after failure to an HMA, HMA-based treatment with guadecitabine can be an effective alternative treatment option prolonging survival in a small proportion of HR-MDS and AML patients, with a toxicity profile similar to that of standard HMA. The authors present an analysis of prognostic factors for response and prolonged OS after guadecitabine treatment. Especially patients with primary azacitidine failure, absence or limited number of somatic mutations and lower methylation level in blood during the first cycle of treatment benefited from guadecitibaine treatment.? A phase III trial comparing guadecitabine with treatment of choice in MDS patients after HMA failure is currently ongoing (clinicaltrials.gov identifier: 02907359).

One other promising approach to improving efficacy of hypomethylation in HR-MDS and AML patients is the addition of the orally selective B-cell lymphoma 2 (BCL-2) inhibitor venetoclax to HMA. BCL-2 protein, a key regulator of leukemic blast survival, has been reported to play an important role in regulating apoptosis via the intrinsic mitochondrial cell death. Overexpression of the BCL-2 protein has been shown to be associated with poor outcomes, conferring chemotherapeutic resistance in AML. ${ }^{8}$ Recent data suggest that $400 \mathrm{mg}$ of venetoclax has an optimal benefit-risk profile when used in combination with azacitidine. ${ }^{8}$ This combination has already demonstrated impressive rates of CR both in the frontline and relapse settings: AML patients treated with first-line venetoclax and HMA showed favorable overall response rates (ORR) (CR: $71 \%$ and CRi: 74\%).9,10 Median duration of response after achieving CR was 21.2 months and median overall survival was 16.9 months. ${ }^{9}$ Comparing these results with

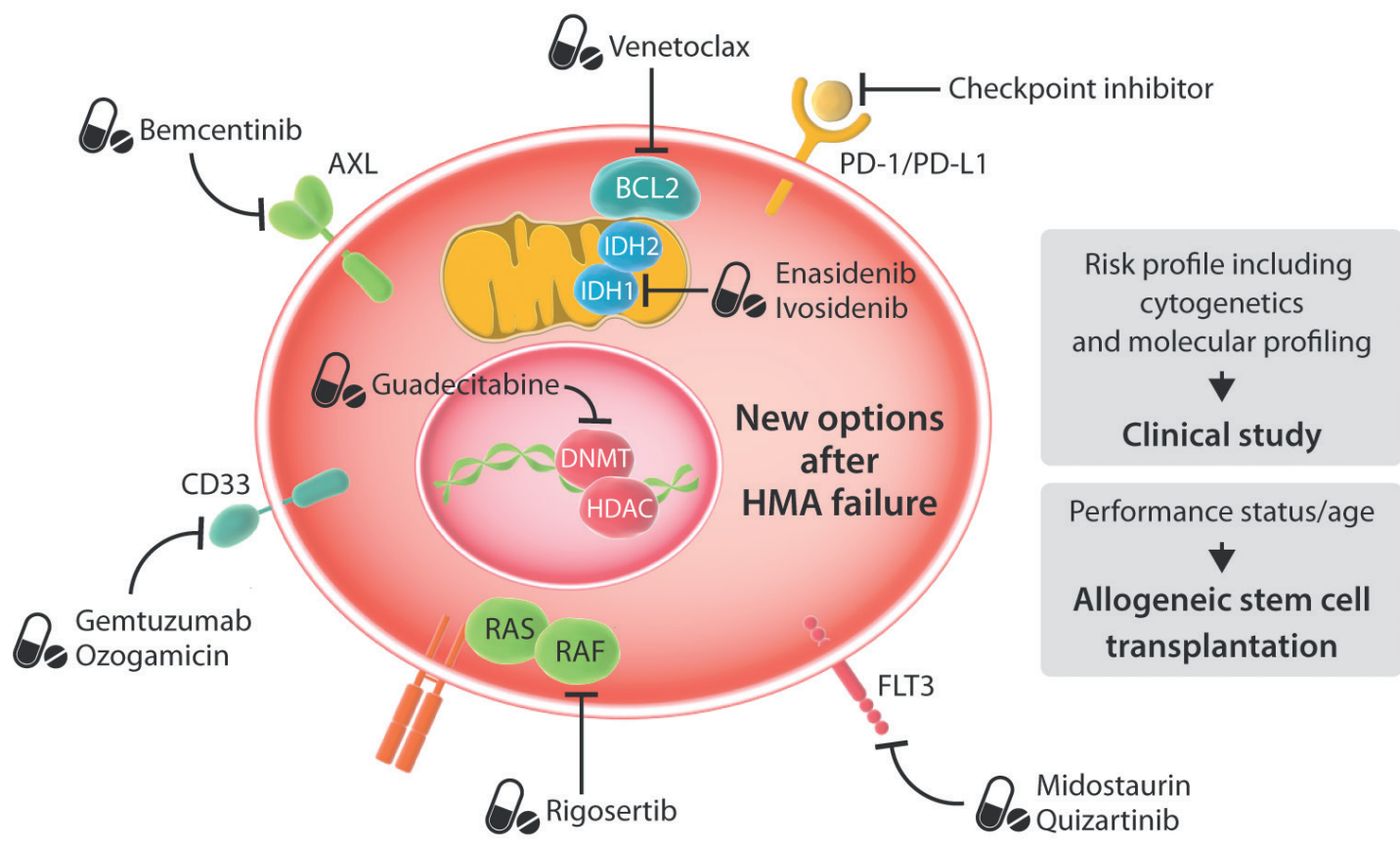

Figure 1. New options after hypomethylating agent failure. 
historically poor outcome data of single agent azacitidine treatment (CR rates approx. 20\%; OS not exceeding 12 months in AML patients ${ }^{3}$ ), it becomes clear that this novel targeted combined strategy will potentially dominate the future treatment landscape in HR-MDS and AML patients not eligible for intensive induction therapy. Nevertheless, previous clinical trials in AML demonstrated a toxicity profile that represents cause for concern. Over $50 \%$ of included patients developed grade $\geq 3$ neutropenia, leading to a high incidence of treatment interruption and subsequent study discontinuation due to progressive disease (PD)., ${ }^{9,11}$ Recruitment is currently underway for a clinical study evaluating the combination of venetoclax with azacitidine in patients with HR-MDS after HMA failure (clinicaltrials.gov identifier: 02966782).

It is known that HMA can reduce immune response by upregulation of inhibitory immune checkpoint molecule expression. Therefore, preventing resistance to HMA by combining HMA and checkpoint inhibition is another possible new treatment strategy which is currently under investigation in several clinical trials. We reported on a patient with sAML undergoing single agent pembrolizumab (anti-PD-1) treatment. ${ }^{12}$ After two months of therapy, platelet count increased in line with a response according to International Working Group (IWG) 2018 criteria, together with clearance of IDH1 mutation. ${ }^{12}$ Recently Daver et al. reported on a phase II study evaluating response to azacitidine and nivolumab in relapsed/refractory AML patients. In HMA pretreated patients, ORR was $22 \%$ and median OS for the 70 included patients was 6.3 months. ${ }^{13}$

Gemtuzumab ozogamicin (GO), a humanized antiCD33 antibody conjugate, is currently licensed by both the US Food and Drug Administration and the European Medicines Agency in combination with daunorubicin and cytarabine for the treatment of de novo CD33-positive AML patients. Moreover, available data suggest activity of GO in combination with HMA. The maturation of AML blasts increases CD33 expression after HMA therapy, resulting in an enhanced uptake of GO by blast cells. ${ }^{14}$ A phase II clinical trial in older AML patients evaluated the combination of hydroxyurea followed by azacitidine for seven days and GO on day eight. Results demonstrated CR in $44 \%$ of patients in the good risk group (age 6069 years or performance status $0-1$ ) and $35 \%$ (19 of 59 patients) CR rate in the poor risk group (age $\geq 70$ years and performance status 2 or 3$) \cdot{ }^{14}$ In a phase II study in newly diagnosed or relapsed HR-MDS and AML patients, the combination of decitabine with GO achieved CR/CRi in $35 \%$ of patients (39 of 110 patients). ${ }^{15}$

Rigosertib (ON-01910), a multikinase inhibitor, is currently undergoing evaluation in a randomized phase III trial (clinicaltrials.gov identifier: 02562443) in HR-MDS patients after HMA failure. Results of a previous phase III study demonstrated that patients treated with rigosertib had longer (8.6 vs. 5.3 months) median OS compared to patients receiving best supportive care after HMA failure. ${ }^{16}$ The combination of rigosertib with azacitidine after HMA failure was recently evaluated in a phase II trial, showing an ORR of $54 \%$, including $8 \% \mathrm{CR}$ in this patient population; the safety profile was similar to those described for azacitidine alone. ${ }^{17}$
One interesting new therapeutic target in patients failing HMA is the selective inhibition of AXL, a surface membrane protein kinase receptor on blast cells. Signaling through AXL seems to stimulate a number of pro-survival pathways and enables malignant cells to develop resistance to conventional chemotherapies. ${ }^{18}$ Preclinical studies with bemcentinib, an orally selective small molecule AXL inhibitor, demonstrated in vitro and in mouse models that leukemic proliferation was blocked by interference with AXL signaling. ${ }^{18}$ Thus, AXL represents a promising target and bemcentinib a possible new treatment option for HR-MDS or AML patients. ${ }^{18}$ The efficacy and safety of bemcentinib is currently being evaluated in a phase II study (BERGAMO trial; clinicaltrials.gov identifier: 03824080) within the European Myelodysplastic Syndromes Cooperative Group (EMSCO) in patients with HR-MDS or AML after HMA failure. Other potentially available therapeutic approaches after failing HMA include the use of targeted molecular therapies, e.g. with IDH or FLT3-inhibitors. IDH mutations are quite common in MDS (10-15\% of patients) and data in relapsed AML have so far proved promising. ${ }^{19}$ FLT3-inhibitors have already been approved in the US for second-line treatment of patients with AML and may, therefore, offer a therapeutic option in rare FLT3 mutated cases with disease progression..$^{20}$

In conclusion, patients with HR-MDS or AML failing HMA remain a population with a dismal outcome and limited therapeutic options. In the future, a personalized targeted treatment strategy on the basis of the patient's molecular profile, cytogenetics, and previous therapies may be the best approach. Until then, translational studies based on a variety of prospective clinical trials are urgently required to overcome the enormous unmet medical need for additional treatment options.

\section{References}

1. Platzbecker U. Treatment of MDS. Blood. 2019;133(10):1096-1107.

2. Fenaux P, Mufti GJ, Hellstrom-Lindberg E, et al. Efficacy of azacitidine compared with that of conventional care regimens in the treatment of higher-risk myelodysplastic syndromes: a randomised, open-label, phase III study. Lancet Oncol. 2009;10(3):223-232.

3. Dombret H, Seymour JF, Butrym A, et al. International phase 3 study of azacitidine vs conventional care regimens in older patients with newly diagnosed AML with $>30 \%$ blasts. Blood. 2015;126(3):291299.

4. Itzykson R, Thepot S, Quesnel B, et al. Prognostic factors for response and overall survival in 282 patients with higher-risk myelodysplastic syndromes treated with azacitidine. Blood. 2011:117(2):403-411

5. Craddock CF, Houlton AE, Quek LS, et al. Outcome of Azacitidine Therapy in Acute Myeloid Leukemia Is not Improved by Concurrent Vorinostat Therapy but Is Predicted by a Diagnostic Molecular Signature. Clin Cancer Res. 2017;23(21):6430-6440.

6. Komrokji RS. Treatment of Higher-Risk Myelodysplastic Syndromes After Failure of Hypomethylating Agents. Clin Lymphoma Myeloma Leuk. 2015;15 Suppl:S56-59.

7. Sebert M, Renneville A, Bally C, et al. A phase II study of guadecitabine in higher-risk myelodysplastic syndrome and low blast count acute myeloid leukemia after azacitidine failure. Haematologica. 2019, 104(8):1565-1571.

8. Dinardo CD, Pratz KW, Potluri J, et al. Durable response with venetoclax in combination with decitabine or azacitadine in elderly patients with acute myeloid leukemia (AML). J Clin Oncol. 2018;36(15 suppl): 7010-7010.

9. DiNardo CD, Pratz K, Pullarkat V, et al. Venetoclax combined with decitabine or azacitidine in treatment-naive, elderly patients with acute myeloid leukemia. Blood. 2019;133(1):7-17. 
10. Pollyea DA, Pratz KW, Jonas BA, et al. Venetoclax in Combination with Hypomethylating Agents Induces Rapid, Deep, and Durable Responses in Patients with AML Ineligible for Intensive Therapy. Blood. 2018;132(Suppl 1):285-285.

11. DiNardo CD, Pratz KW, Letai A, et al. Safety and preliminary efficacy of venetoclax with decitabine or azacitidine in elderly patients with previously untreated acute myeloid leukaemia: a non-randomised, open-label, phase 1b study. Lancet Oncol. 2018;19(2):216228 .

12. Kubasch AS, Wehner R, Bazzurri S, et al. Clinical, molecular, and immunological responses to pembrolizumab treatment of synchronous melanoma and acute myeloid leukemia. Blood Adv. 2018;2(11):1187-1190.

13. Daver N, Garcia-Manero G, Basu S, et al. Efficacy, Safety, and Biomarkers of Response to Azacitidine and Nivolumab in Relapsed/Refractory Acute Myeloid Leukemia: A Nonrandomized, Open-Label, Phase II Study. Cancer Discov. 2019;9(3):370-383.

14. Nand S, Othus M, Godwin JE, et al. A phase 2 trial of azacitidine and gemtuzumab ozogamicin therapy in older patients with acute myeloid leukemia. Blood, 2013;122(20):3432-3439.

15. Daver N, Kantarian H, Ravandi F, et al. A phase II study of decitabine and gemtuzumab ozogamicin in newly diagnosed and relapsed acute myeloid leukemia and high-risk myelodysplastic syndrome. Leukemia. 2016;30(2):268-273.

16. Garcia-Manero G, Fenaux P, Al-Kali A, et al; ONTIME study investigators. Rigosertib ver- sus best supportive care for patients with high- risk myelodysplastic syndromes after failure of hypomethylating drugs (ONTIME): a rando- mised, controlled, phase 3 trial Lancet Oncol. 2016;17(4):496-508.

17. Navada SC, Garcia-Manero G, Atallah EL, et al. Phase 2 Expansion Study of Oral Rigosertib Combined with Azacitidine (AZA) in Patients (Pts) with Higher-Risk (HR) Myelodysplastic Syndromes (MDS) Efficacy and Safety Results in HMA Treatment Naïve \& Relapsed (Rel)/Refractory (Ref) Patients. Blood. 2018;132(Suppl 1):230.

18. Medyouf $\mathrm{H}$. The microenvironment in human myeloid malignan cies: emerging concepts and therapeutic implications. Blood. 2017;129(12): 1617-1626.

19. Stein EM, DiNardo CD, Pollyea DA, et al. Enasidenib in mutant IDH2 relapsed or refractory acute myeloid leukemia. Blood. 2017;130(6):722-731.

20. Cortes J, Perl AE, Döhner H, et al. Quizartinib, an FLT3 inhibitor, as monotherapy in patients with relapsed or refractory acute myeloid leukaemia: an open-label, multicentre, single-arm, phase 2 trial Lancet Oncol. 2018;19(7):889-903.

\section{Discontinuation of tyrosine kinase inhibitors in patients with chronic myelogeneous leukemia - You can do this at home if you read the instructions}

\section{Charles A. Schiffer}

Joseph Dresner Chair for Hematologic Malignancies, Departments of Oncology and Medicine, Wayne State University School of Medicine, Karmanos Cancer Institute, Detroit, MI, USA

E-mail: CHARLES A. SCHIFFER - Schiffer@karmanos.org

doi:10.3324/haematol.2019.222216

T he exciting story of the clinical use of imatinib mesylate for the treatment of leukemias driven by the bcr/abl mutation began in the late 1990s and dramatic effectiveness was immediately apparent in all stages of the diseases. Although there was concern that these benefits might not persist, we now know, after almost twenty years of follow up, that a high proportion of chronic phase patients attain deep molecular responses and enjoy an overall survival comparable to that of agematched controls. ${ }^{1}$ It was originally expected that lifelong treatment would be needed, but in recent years, trials from around the world have shown that tyrosine kinase inhibitors (TKI) can be successfully discontinued in some patients who have achieved sustained deep molecular responses. ${ }^{2,3}$

These were conducted as part of clinical trials at CML research institutions by experienced CML clinicians. In this issue of the Journal, Italian clinicians from a wide range of institutions of the Gruppo Italiano Malattie Ematologiche dell'Adulto (GIMEMA) describe a large group of chronic phase patients who had therapy discontinued, many presumably as a consequence of patients' requests to doctors, who were now comfortable with the accumulated results. ${ }^{4}$ With a median follow up of 34 months, $60 \%$ of patients remained in what has been termed "treatment-free remission" (TFR), ${ }^{5}$ a result consistent with or perhaps slightly superior to those from earlier trials. As in other trials, the relapse rate was somewhat lower in patients with longer exposures to TKI and all patients who had molecular relapse were successfully retreated with either their original TKI or were switched to another TKI if their motivation for discontinuation was toxicity; these retreated patients usually achieved the level of their original response.

Most CML patients in the US (and to some extent elsewhere) are not followed in specialty hematology centers. This means that the next question in the TKI saga is whether discontinuation can be managed safely by nonspecialist oncologists. The process is not very difficult to understand and there are few risks if patients are selected and followed appropriately. The criteria for study entry and monitoring differed somewhat amongst the published trials, but a consensus approximation would include:

- TKI treatment for a minimum of three years;

- continuous deep molecular response [minimum MR4 (BCR-ABL1 $\leq 0.01 \%$ using the International Scale, IS)] on multiple testing for at least two years. Some studies required reduction to $<M R 4.5$, although outcomes seem comparable (including the results from this GIMEMA experience) using either molecular cutoff;

- use of a quantitative polymerase chain reaction (qPCR) test sensitive to a level of at least MR4.5 in a laboratory with a rapid turn-around time;

- monitoring of peripheral blood transcripts every 4-6 weeks for 6-8 months, then bimonthly for approximately one year followed by every three months thereafter for a minimal follow up of three years; 